\title{
THE DESIGN AND APPLICATION OF THE SELF-ADAPTIVE AUTOMATIC CONTROL SHEARER DRUM
}

\author{
Guoqiang GENG \\ Henan University of Engineering, Henan, China \\ Email: gengguoqiangfly@126.com
}

\begin{abstract}
In order to solve the current situation that under geological change, the shearer cannot self-adaptively adjust to the memory of the cutting track, thereby reducing the efficiency of the coal shearer, an adaptive 3D fine geologic model based on the method of complex geological structures is proposed. In the study, a mathematical model of the cutting trajectory of the shearer drum is set up by three coordinate transformations, and the relatively high precision of the cutting trajectory of the shearer drum is obtained. Then, the circular coordinate transformation method is used to plan the cutting path of the shearer, and the calculation formula of the adjustment and the angle of the pitch angle for every shearer of the shearer is derived. In the end, according to the requirements of the mining technology of the shearer, the correction method of the theoretical planning path of the shearer based on the interpolation algorithm and the cyclic coordinate transformation algorithm is put forward. The research shows that the method proposed in this paper can not only adapt to complicated geological structures such as folds, but it also can achieve greater recovery rate.
\end{abstract}

Keywords: Coal shearer; Self-adaptive; Interpolation algorithm; Cyclic coordinate transformation algorithm; Complex geological structure.

\section{Introduction}

The shearer is one of the main equipment for the full mechanization of combined coal mining face. Double Drum Shearers have become the mainstream shearers in fully mechanized coal mining face.

Whether the Two Drum Shearers can work safely and reliably has become a growing concern. The automatic height adjustment technology of the shearer is an important part of the realization of the automation working face [1]. With the exception of a handful of foreign enterprises, the adjustment of shearer drum height in a majority of companies still adopts the manual control. The manual control is, specifically, by visual observation and the noise of and cutting, the shearer drivers use his experience to determine whether it is cutting coal or rock cutting in order to adjust the vertical position of the drum. If the position adjustment of the drum is too slight, it will cause too much surplus on the top and reduce the recovery rate, resulting in a great waste of resources. At the same time, the manual operation of the shearer driver may cause a series of problems, such as the uneven surface of the roof and floor, resulting in difficulties in shifting and pushing.

Therefore, how to realize the automatic height adjustment of the shearer drum is of great significance to the improvement of the level of the equipment of the working face.

\section{Literature Review}

At present, in view of the adaptive control of the shearer, two kinds of automatic height adjustment technology for coal shearers are put forward by domestic and foreign scholars. One is the direct height adjustment method based on the identification of the coal and rock interface of the sensor, and the other is the indirect height adjustment technology based on memory cutting [2].

However, these two methods are not good enough to adapt to the complex geological environment with significant changes in coal mines and have a low recovery rate, bringing serious error and high cost in the process of coal cutting. Even though many domestic and foreign scholars put forward using dynamic fuzzy neural network (DFNN), generalized regression neural network (GRNN), grey Markov model of shearer along the feed direction of the mining height adjustment to forecast the quantity, but because of the shearer working environment is complex and the uncertainty of the geological environment, the application can barely be put into actual use. Therefore, based on the memory cutting technology, this study proposes the controlling idea of automatically correcting the memory cutting path by using the three-dimensional refined geological database, hoping to provide reference for the adaptive technology of shearers in practical applications. 


\section{Methodology}

3.1 The establishment of a mathematical model for the cutting trajectory of a coal shearer

Due to the large volume of the shearer body and the relative rotation between the left and right rocker and the body of the shearer, it cannot be regarded as a particle. Therefore, a reference point must be selected to represent the location relationship of each part of the shearer. In order to accurately indicate the location of the shearer's left and right rollers, this paper selects the centre of mass of the shearer as the reference point, establishes a coordinate system fixed to the shearer, and provides a rule for the coordinate system [3].

The original point $\mathrm{Ob}$ of shearer coordinate system coincides with the centre of mass of the shearer, $\mathrm{Xb}$ direction is perpendicular to the direction of the shearer body, $\mathrm{Yb}$ direction is perpendicular to the direction of the shearer body, and the $\mathrm{Zb}$ direction is perpendicular to the shearer body.

The real time position and heading of shearer are measured by gyroscope in inertial navigation system. The swing angle of shearer rocker is measured by rotary encoder, and the position of shearer fuselage can be obtained by combining inertial navigation system [4]. Shearer position angle and yaw angle can accurately show the relationship between positions in a moment of shearer ObXbYbZb coordinate system and the geographic coordinate system OtXtYtZt; rocker

$$
R_{t}^{b}=\left[\begin{array}{ccc}
\cos \gamma & 0 & -\sin \gamma \\
0 & 1 & 0 \\
\sin \gamma & 0 & \cos \gamma
\end{array}\right] \times\left[\begin{array}{ccc}
1 & 0 & 0 \\
0 & \cos \beta & \sin \beta \\
0 & -\sin \beta & \cos \beta
\end{array}\right] \times\left[\begin{array}{ccc}
\cos \delta & \sin \delta & 0 \\
-\sin \delta & \cos \delta & 0 \\
0 & 0 & 1
\end{array}\right]
$$

The position coordinates of the shearer drum under the local geographic coordinate system of the mining area can be expressed as:

$$
P_{i}^{g}=P_{0}^{g}+R_{b}^{t} \times P_{i}^{b}(i=1,2)
$$

The cutting trajectories of the shearer in real time are obtained as shown in Figure 1 and Figure 2.

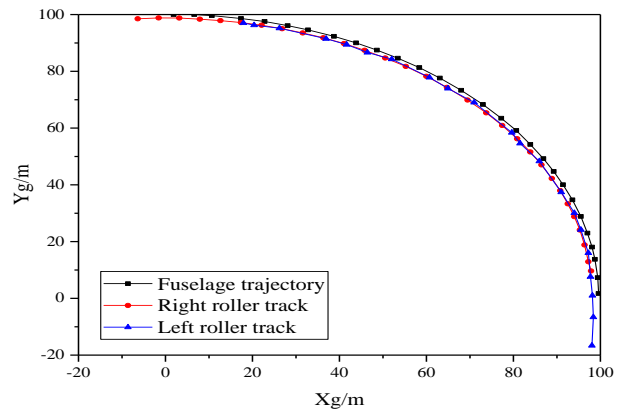

Figure 1: The motion track of the fuselage and the roller when the fuselage of a coal shearer does circular arc motion rotary encoder can accurately represent a time fuselage and shearer rocker arm relative pose between shearer machine body position; the shearer's position can accurately show the relationship between geographic location coordinate with OtXtYtZt local mining geographic coordinate system OgXgYgZg.

After determining the relative positions of the above, the principle of inertial navigation is adopted, and through the coordinate transformation method three-dimensional coordinate transformation between the coordinate system of shearer and ObXbYbZb mining local geographic coordinate system OgXgYgZg can be realized. Then you can have the trajectory of Shearer in local geographic coordinates [5].

There is a relative rotation between the rocker arm and the fuselage of the shearer, so the coal shearer coordinate system can be expressed as:

$$
\begin{aligned}
& P_{1}^{b}=\left[\begin{array}{c}
B \\
l / 2 \\
0
\end{array}\right]+\left[\begin{array}{ccc}
0 & 0 & 0 \\
0 & \cos \theta_{1} & 0 \\
0 & \sin \theta_{2} & 0
\end{array}\right] \times\left[\begin{array}{c}
0 \\
L_{0} \\
0
\end{array}\right] \\
& P_{2}^{b}=\left[\begin{array}{c}
B \\
-l / 2 \\
0
\end{array}\right]+\left[\begin{array}{ccc}
0 & 0 & 0 \\
0 & \cos \left(-\theta_{1}\right) & 0 \\
0 & \sin \left(-\theta_{2}\right) & 0
\end{array}\right] \times\left[\begin{array}{c}
0 \\
-L_{0} \\
0
\end{array}\right]
\end{aligned}
$$

The coordinate transformation matrix of the coal shearer coordinate system is:

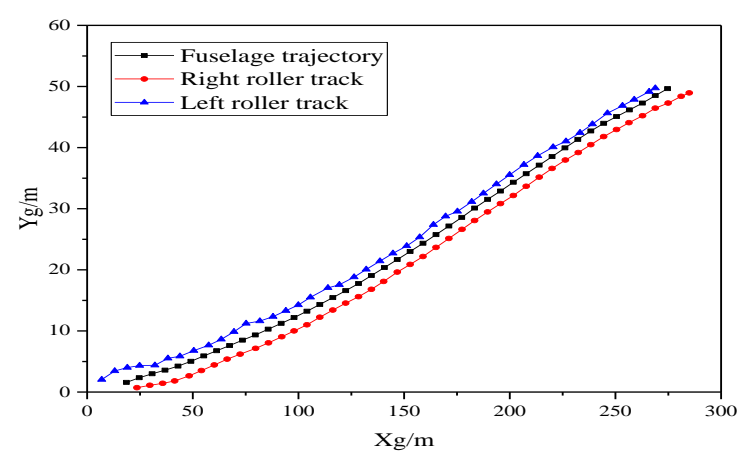

Figure 2: Cutting trajectory of a shearer along the direction of traction

\subsection{Adaptive cutting path planning for fold geological structure}

The process of mathematical description of continuous geological structure (folds) is to read the coordinate values of the top and bottom coal and 
rock interface from the three-dimensional refined geological model, and obtain the occurrence of continuous geological structure (fold) by three times spine interpolation [6]. The top and bottom curve obtained by the interpolation operation is the reference cutting path under the condition of continuous geological structure (fold) [7]. The interpolation algorithm is defined as follows.

Assume that function $\mathrm{y}=\mathrm{f}(\mathrm{x})$ is defined in the interval $[a, b]$, and it is known that at $a \leq x 0 \leq \ldots \leq x n \leq b$, the value isf(xi)=yi $(\mathrm{i}=0,1, \ldots, \mathrm{n})$, if there is a simple function, $\varphi(\mathrm{x})$, so that:

$$
\varphi\left(x_{i}\right)=y_{i}(i=0,1,2, \ldots, n)
$$

It is established that $\varphi(\mathrm{x})$ is an interpolating function of $f(x)$, point $X i(i=1,2, \ldots N)$ is called an interpolated node. $\varphi(\mathrm{x})$ is an algebraic polynomial of no more than $\mathrm{N}$ :

$$
\varphi_{n}(x)=a_{0}+a_{1} x+\ldots+a_{n} x^{n}
$$

In which, $a_{i}(i=1,2, \ldots N)$ is a real number. $\varphi_{n}(x)$ is an interpolating polynomial.

In the process of data processing, the three-spine interpolation algorithm has been widely applied, and has been proved to have good performance. Compared with other fitting algorithms, the threeorder spine interpolation showed good two-order smoothness [8]. In carrying out the study, along the feed direction of coal mining machine $\mathrm{X}=0 \mathrm{~m}$, $\mathrm{Y}=1500 \mathrm{~m} \sim 1800 \mathrm{~m}$ coordinates of the seam roof and floor, from the Shanxi Xiegou Coal Mine acquired mining area, the three-spine curve of roof and floor of the geological structure under the condition of fold interpolation by bottom curve of coal seam roof is worked out, as shown in figure 3.

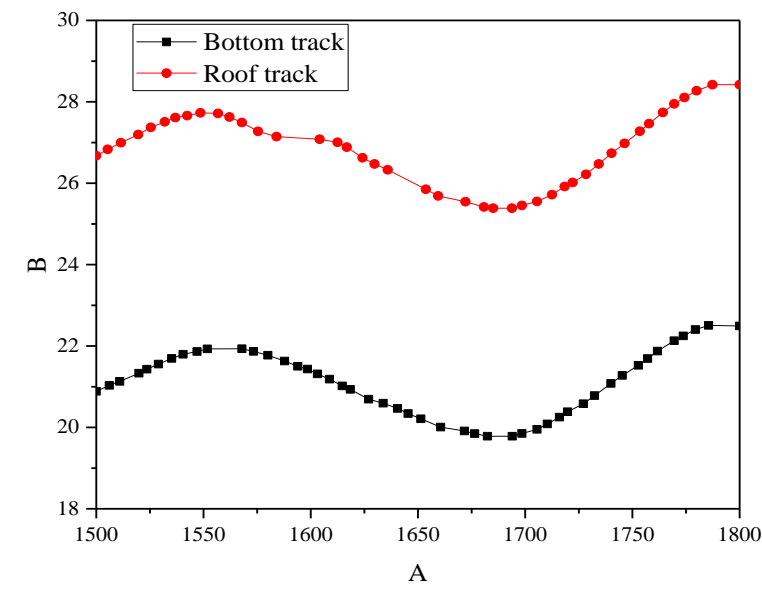

Figure 3: The curve of top and bottom of coal seam based on three-spine interpolation

In order to achieve the maximum recovery rate, the path planning method along the cutting direction of the shearer is analyzed with the anticline in the fold geological structure as an example. In order to achieve this goal, under the condition of fold geological structure, the shearer only needs to cut the interface curve of the top and bottom coal and rock along the interpolation operation. Through the folds geological structure along the feed direction, the coal mining machine must adjust the bottom roller of the shearer gradually in order to adapt to the change of coal seam floor [9].

Under the geological condition of the anticline, the 0 point is the point where the development of the folded geological structure begins.

The lower drum of the shearer cuts to the shearer's feed direction once, but it doesn't make adjustments to its undercover volume. The second feed is called the zeroth knife. After cutting zeroth knives, the angle between the pushing and sliding moving of the fully mechanized mining equipment and the feed direction of the shearer is 0 degrees. The angle between them is the same as that of $\gamma 0$. The shearer advances a depth of $\mathrm{d} 1$ along the direction of the pitch angle $\gamma 0$, adjusts the undercover of the cutting drum, and the adjustment is recorded as $\mathrm{T} 1$. When the shearer completes the action of feed and height adjustment, the fully mechanized mining equipment will carry out a push and slide process along the feed direction in the direction of 0 to 1 . The shearer again feed, the dip with a knife "push mining equipment" and "dip shift frame" are the same with the previous one. Shearer is again adjusted and completed undercover. The $1 \rightarrow 2$ directions of the "push" and "moving" process between shearer and feed direction of fully mechanized mining equipment in the "push" and "moving" direction of the value is 1 , the coal mining machine under the knife of the upward mining angle is $\gamma 0+\varphi 1$. The coal mining machine can automatically plan the cutting path under the geological structure of the anticline.

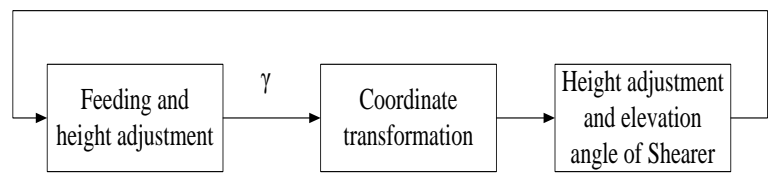

Figure 4: The calculation process of the folds of the shearer

Figure 4 shows the calculation process of shearer passing through fold. It shows that after the mining machine completed a feed and increase, the angle $\varphi_{i}$ between feed direction of the shearer and the direction of fully mechanized mining equipment "push" and "moving forward" can be calculated. $\varphi_{\mathrm{i}}$ is the angle between feed directions before and after the cutter shearer.

Then the coordinate transformation is carried out to calculate the height of the cutter and the angle of the pitching mining to guide the process of the next cycle [10]. In order to more intuitively represent the path planning process of shearers based on the curve of roof and floor, according to the coordinate 
transformation principle, we use formula (7) to calculate the adjustment amount of every cutter of shearers.

$$
\begin{array}{r}
{\left[\begin{array}{l}
Y_{i} \\
Z_{i}
\end{array}\right]=T_{i} \times\left[\begin{array}{l}
Y \\
Z
\end{array}\right]} \\
T_{i}=\left[\begin{array}{cc}
\cos \varphi_{i} & \sin \varphi_{i} \\
-\sin \varphi_{i} & \cos \varphi_{i}
\end{array}\right]
\end{array}
$$

\subsection{Planning of sectional cutting path of fault geological structure}

When the shearer moves along the feed direction of the fault zone, first obtain the geological parameters of fault tectonic from the 3D geological model of engineering geological exploration and seismic exploration based on CT, and the use these parameters to accurately expressed the characteristic of fault geological structure along the feed direction of the shearer; based on manual adjustment, to see if shearer can adapt to fault zone using coal mining technology to define for itself the fault vertical drop $h$ and fault zone influence range $L$.

If the above conditions are satisfied, the shearers can go through the fault zone adaptively through path planning and the correction of planning path based on interpolation algorithm. If these conditions do not meet the above requirements, it is necessary to manually adjust the way through the fault zone [11].

The starting point $(A, C)$ and end point $(B, D)$ of cutting path of coal mining machine feed direction, the upper and lower plate transition section of shearer can be determined through the influence range $\mathrm{L}$ of the fault zone and the basic parameters of the fault zone.

Assuming the shearer through fault belt along the feed direction of the shearer length is L, the time the amount of feed is D, because the pitch of Shearer in process of the fault zone in the coal mining machine mining point will change, it is difficult to determine the number of feed through the length of the $L$ fault zone [12].

In addition, assuming that the shearer's cutting direction is the same as that of the shearer before passing through the fault, the number of times for the Shearer to go through the fault zone is $\mathrm{N}=\mathrm{L} / \mathrm{d}$, and the maximum adjustment amount of the shearer cutting height is $\mathrm{h}$ in the adjustment process.

In order to ensure the smooth movement of fully mechanized mining equipment "moving frame" and "pushing sliding" when the shearer passes through the fault zone, we must ensure the continuity of geological conditions during the cutting process, that is, the difference between the two cutting heights before and after cutting is less than $0.15 \mathrm{~m}$, that is:

$$
\frac{h}{L / d} \leq 0.15
$$

In view of the need to adjust the cutting height or the bottom of the shearer before the shearer reaches the fault zone of the working face, it should be guaranteed:

$$
L \geq G H \times \cos (\alpha+\varphi)
$$

$\mathrm{GH}$ is the overlap line of fault section.

In order to improve the working efficiency of the shearer in the process of passing through the fault zone and reduce the number of the cutting tools as much as possible, the value of $L$ is set:

$$
L=\max \{h \times d / 0.15, G H \times \cos (\alpha+\varphi)\}
$$

In order to ensure the starting point of the cutting path of the transition section is on the top floor of the coal seam of the fault zone, the range of the value of the $\lambda$ is as follows:

$$
O G \times \sin (\alpha+\varphi) / L \leq \lambda \leq 1
$$

The following two constraints must be taken into account if the coal shearer adaptively passes the fault zone on the premise of realizing the minimum residual amount of triangulation and the minimum cut rock quantity. The two constraints can be used to verify the reliability of the planned cutting path to determine whether the shearer can pass through the fault zone normally. The two constraints are the passing capacity and continuity of the equipment respectively [13].

The capacity of the equipment is an important factor in determining whether the coal shearer can realize the automatic pass over fault. Whether the equipment can safely and reliably pass through the fault belt is mainly related to the distance between the cutting trajectory of the upper and lower rollers of the fault belt of the shearer. If the shearer can pass the fault belt smoothly, it is necessary to ensure that the distance between the top and lower cutting tracks of the shearer along the cutting direction of the shearer is greater than the maximum height of the fully mechanized coal mining equipment.

The two cutting trajectories obtained from the above calculation are parallel to each other, and the distance between the two cutting trajectories is calculated by the formula (13).

$$
c=\left|c_{1}-c_{2}\right| / \sqrt{a^{2}+b^{2}}
$$

The maximum height of the equipment is the diameter of the shearer drum, the maximum value of the combination of: the minimum height of the support, the maximum height of the shearer's fuselage and the thickness of the top beam of the 
frame. Therefore, the constraint condition can be expressed by formula as (14):

$$
c \geq \max \left\{D, h^{\prime}+h^{\prime \prime}, h_{z}+0.3\right\}
$$

The continuity requirement refers to the continuity of the two- cutting trajectory of the shearer before and after, which is height difference of two sampling points along the feed direction of the shearer (above or below) before and after the cutting trace. It is also the requirement of maximum amount of adjustment along the feed direction before and after the shearer cutting height [14].

According to the experience of the manual control of the shearer's over fault, the adjustment of the shearer must not be more than $0.15 \mathrm{~m}$. Use of expression (15) to judge, if it can meet the requirements, then the path meets the continuity requirements; otherwise not.

$$
0.15 / d \geq-a / b
$$

The path correction process in the fault zone for the shearer is shown in Figure 5.

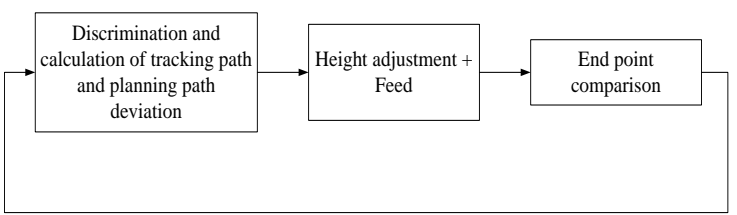

Figure 5: Fault zone cutting path correction process based on interpolation algorithm

\section{Results and Discussion}

\subsection{Error analysis of 3-dimension position coordinate of shearer drum}

The absolute position error of the shearer drum can be expressed as:

$$
\Delta P_{i}^{g}=\left[\left|\frac{\partial P_{i}^{g}}{\partial \delta}\right|\left|\frac{\partial P_{i}^{g}}{\partial \beta}\right|+\left|\frac{\partial P_{i}^{g}}{\partial \gamma}\right|\left|\frac{\partial P_{i}^{g}}{\partial \theta_{i}}\right|\right]\left[\begin{array}{c}
\Delta \delta \\
\Delta \beta \\
\Delta \theta_{i}
\end{array}\right]
$$

The error permissible range of the required sensors is combined with the error factor matrix $\mathrm{K}$, then you have the following:

$$
K_{i}^{-1}\left[\begin{array}{c}
-\Delta X_{i}^{g} \\
-\Delta Y_{i}^{g} \\
-\Delta Z_{i}^{g}
\end{array}\right] \leq\left[\begin{array}{c}
\Delta \delta \\
\Delta \beta \\
\Delta \theta_{i}
\end{array}\right] \leq K_{i}^{-1}\left[\begin{array}{c}
\Delta X_{i}^{g} \\
\Delta Y_{i}^{g} \\
\Delta Z_{i}^{g}
\end{array}\right](i=1,2)
$$

Following the above calculation of the range of the allowable error of the sensor, it can be analyzed:

The parameters in Table 1 are the parameters of the genetic algorithm used to calculate the maximum value of the error factor. From a Shanxi coal mine geological exploration data and seismic wave CT detection, fine geophysical data is obtained, which shows that delta $\delta \in\left[89^{\circ}, 91^{\circ}\right], \beta \in\left[9^{\circ}, 11^{\circ}\right]$, $\gamma \in\left[-5^{\circ}, 5^{\circ}\right]$.

The range of values of these variables is considered as a constraint in the calculation process of genetic algorithm.

Table 1 Parameters in genetic algorithm

\begin{tabular}{|c|c|c|c|}
\hline $\begin{array}{l}\text { Population } \\
\text { number }\end{array}$ & $\begin{array}{l}\text { Maximum } \\
\text { iteration }\end{array}$ & $\begin{array}{l}\text { Crossover } \\
\text { probability }\end{array}$ & $\begin{array}{l}\text { Mutation } \\
\text { probability }\end{array}$ \\
\hline 20 & 100 & 0,5 & 0,01 \\
\hline
\end{tabular}

Select three types of shearers working in thin coal seam, medium thick coal seam and thick coal seam: MG80/200-BW, MG132/320-W and MG1000/2660-WD, to study the solving process of the sensor's allowable error range. The required parameters are shown in Table 2. Suppose the three types of shearers work in a coal seam environment with the same $\operatorname{dip}(\beta)$ and strike pitch $(\gamma)$, and ensuring that the range of the heading angle of the shearer is the same.

The permissible range of the cutting trajectory error of the same shearer is given in formula (7).

$$
\Delta P_{i}^{g}=\left[\begin{array}{lll}
0.01 & 0.01 & 0.01
\end{array}\right]^{T}(i=1,2)
$$

Table 2 Parameters of all types of coal cutter

\begin{tabular}{|l|l|l|l|l|l|}
\hline Type & $\mathrm{B}(\mathrm{m})$ & $\mathrm{L}_{0}(\mathrm{~m})$ & $\mathrm{l}(\mathrm{m})$ & Swing range $\left(^{\circ}\right)$ & Seam type \\
\hline MG80/200-BW & 1.1 & 1.40 & 3.7 & {$[-13.1,32.6]$} & Thin coal seam \\
\hline MG132/320-W & 1.3 & 1.71 & 5.6 & {$[-15.8,19.5]$} & Medium-thickness coal seam \\
\hline MG1000/2660-WD & 1.7 & 3.53 & 9.81 & {$[-17,51]$} & Thick coal seam \\
\hline
\end{tabular}

We can see from Figure 6, the thin coal seam shearer's swing range is higher than that of the inertial navigation system for shearer heading and position measurement; and for the thick coal seam and thick coal seam, the pitch angle measurement of inertial navigation system of shearers needs to be more precise than that of the other three parameters.

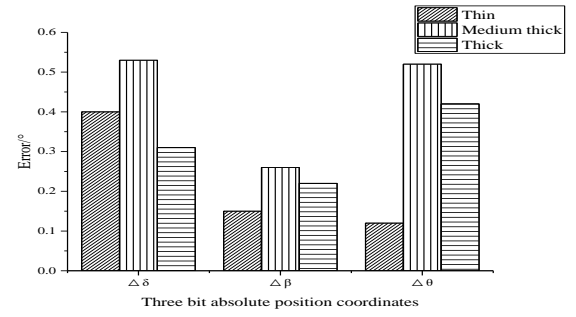

Figure 6: The acceptable error range of the sensors used for three shearers 


\subsection{Simulation analysis of the path planning method of the coal seam floor by the lower drum of the shearer}

Under the condition of the fold geological structure, the path planning method of the coal seam floor is simulated and analyzed by the lower drum of the shearer. First, use simulation data to make a coal seam floor curve. The equation of this curve is $\mathrm{Z}=-$ $0.01 \mathrm{Y}^{2}+0.2 \mathrm{Y}$, the interval range is $[0 \mathrm{~m}, 20 \mathrm{~m}]$, and the cubic term coefficient is 0 . The path planning method for the bottom plate of Figure 7 is planned by using the path planning method of the fold floor.

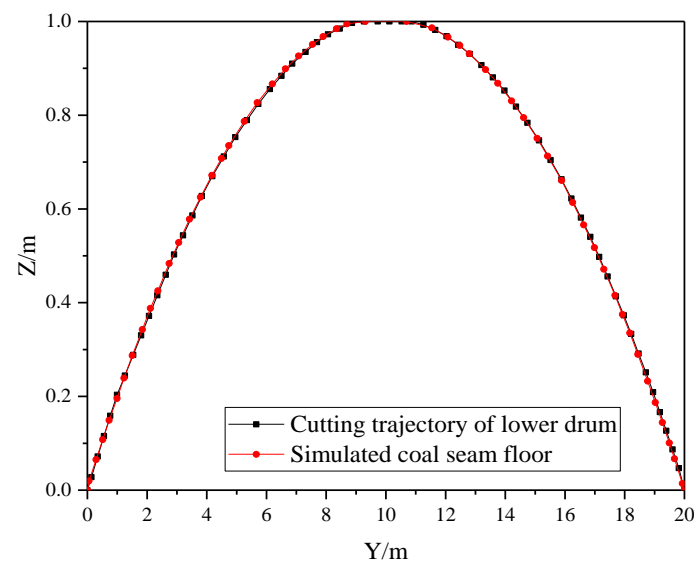

Figure 7: The curve of coal seam floor and its planning path under fold

Figure 7 compared the roof curve and planned path, suggesting that the coal mining machine path planning and the bottom curve has a maximum difference of $0.038 \mathrm{~m}$. This indicates that the shearer drum cutting path planning can reliably pass through the folds of geological structure, at the same time, it can guarantee a large recovery rate and smaller rock cutting amount.

Coal seam floor curve given the above is a continuous curve with a given equation curve. The shearer's adaptive cyclic coordinate transformation over fold geological structure obtained along the feed direction of the cutting path planning is based on a series of line which composed of each segment, the process is of similar mathematics and calculus principle to that of the straight lines". The broken line is as close as possible to the curve of the given coal seam floor, so as to achieve the maximum recovery rate (minimum cutting amount) of the shearer theoretically. Because the amount of feed and the height of the coal cutter in each direction along the feed direction is smaller than that in a long distance, so the theoretical cutting track is basically consistent with the given bottom plate curve.

\subsection{Experiment of coal mining machine through fold under three dimensional geological conditions}

Simulate a fold geological structure in the experimental process that matches a shearer prototype; then use the cyclic coordinate transformation algorithm to calculate the data of feed direction of shearer drum prototype under the mining height (adjusted by undercover volume); leverage the memory cutting algorithm together with geological information on the roof traction for shearer prototype roller adjustment.

The geological structure of a coal seam with a thickness of about $6 \mathrm{~m}$, a fold span of $10 \mathrm{~m}$ and a center elevation of $0.25 \mathrm{~m}$ at the center of the floor is reduced to $1: 10$ in scale. In the simulation, shearer works under the condition of simulated fold geological structure and completes traction movement along the Xg direction. In the process of traction, store the shearer cutting trajectory memory for the initial cutting; according to the simulation of coal seam roof coordinate, adjust its cutting track; after every movement traction shearer, a drum prototype adjustment of the height and pitch angle adjustment are made. The experimental platform cannot complete the real mining equipment with "push" and "moving" function, so the assumption here is that the push conveyor quantity (feed) of coal mining machine is a constant $\mathrm{d}=0.1 \mathrm{~m}$, coal mining machine prototype adjusts the components in the Yg and $\mathrm{Zg}$ direction of each knife feed direction. The cutting process of each cutter using the shearer prototype in the direction of traction in the fold geological structure is as shown in Figure 8.

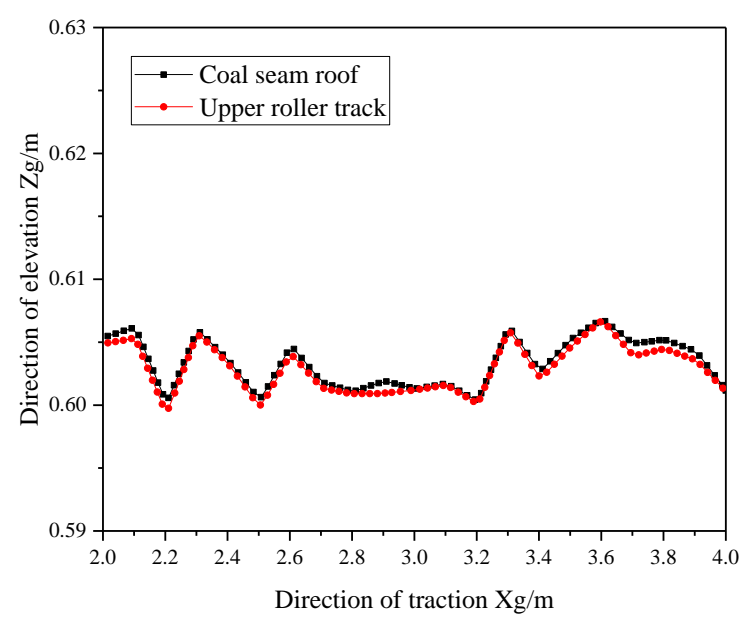

Figure 8: The cutting trajectory of the shearer following the pulling direction under the condition of the fold geological structure when $\mathrm{Yg}=1.0$ 


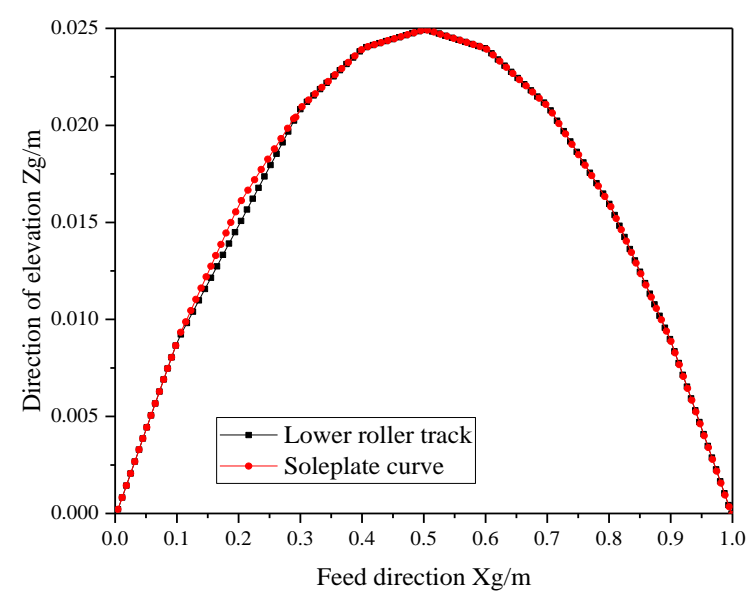

Figure 9: The trajectory of a shearer under the direction of the cut in the fold

In the direction of the mining machine, the cutting trajectories of the upper and lower shearers are shown in figure 7 and 8 respectively. Due to the limitation of the accuracy of the shearer prototype's vibration and swing angle during the experiment, there is a certain deviation between the actual cutting trajectory of the shearer prototype and the curve of the top and bottom under the fold.

According to figure 7 and 8, the maximum height adjustment error of the shearer is $0.0028 \mathrm{~m}$, and the maximum error is $0.47 \%$. Theoretically, the shearer can cut along the top (bottom) plate on the actual working face, but in the same environment as the laboratory, the shearer prototype is reduced by 10 times, and the actual coal mining machine cutting deviation is $0.028 \mathrm{~m}$. The thickness of the coal seam in the actual mining area is $6 \mathrm{~m}$, so the height adjustment error of the shearer is $0.47 \%$ under the real geological environment. At present, the error of the shearer height raised by the related researchers is generally less than $0.06 \mathrm{~m}$. Compared with the result, the accuracy of the shearer is greatly improved by this research method. Therefore, compared with previous studies, this research method can not only adapt environment to pass through complex geological structures such as folds, but it also helps achieve larger recovery rate.

\section{Conclusion}

In this paper, an adaptive control method of shearer based on three-dimensional fine geological model and memory cutting is proposed. First, it proposed the absolute position of shearer drum method, simulation results show that the thin coal seam shearer has higher requirements for swing range than for the inertial navigation system of shearer heading and position measurement; and the middle to thick coal seam shearers have higher requirements for pitching angle measurement of inertial navigation system than the other three parameters. Then the cutting path planning is studied under the cutting direction of the shearer and the geological structure of the fault.

The simulation results show that the maximum height difference between the cut path and the bottom of the floor is $0.038 \mathrm{~m}$. Finally, the experiment of shearer's geological structure under three-dimensional geology is simulated. The experiment shows that the adaptive adjustment method of shearer drum proposed in this paper can not only adapt itself to pass through complicated geological structures such as folds, but it can also achieve higher recovery rate.

\section{References}

[1] Serpell M, Smith JE. (2014). Self-Adaption of Mutation Operator and Probability for Permutation Representations in Genetic Algorithms. Evolutionary Computation, 18 (3), 491-514.

[2] Hao L, LüF, Li L, et al. (2013). Self-adaption of methane-producing communities to $\mathrm{pH}$ disturbance at different acetate concentrations by shifting pathways and population interaction. Bioresource Technology, 140 (3), 319-327.

[3] Shi T, Wang Z, Xia C. (2015). Speed Measurement Error Suppression for PMSM Control System Using Self-Adaption Kalman Observer. IEEE Transactions on Industrial Electronics, 62 (5), 2753-2763.

[4] Xu F, Zhang R, Wu L, et al. (2012). Self-adaption BP neural network for the maximum-powerpoint tracking in photovoltaic. Acta Energiae Solaris Sinica, 33 (3), 468-472.

[5] Su Y, Lu N. (2015). Supply Chain Finance Credit Risk Evaluation Method Based on Self-Adaption Weight. Journal of Computer \& Communications, 03 (7), 13-21.

[6] Yousefi R, Nourani M, Ostadabbas S, et al. (2014). A motion-tolerant adaptive algorithm for wearable photoplethysmographic biosensors. IEEE Journal of Biomedical \& Health Informatics, 18 (2),670.

[7] Xing M, Xiang S, Cai L. (2014). A Real-Time Adaptive Algorithm for Video Streaming over Multiple Wireless Access Networks. IEEE Journal on Selected Areas in Communications, 32 (4),795-805.

[8] Xu GF, Bose T, Kober W, et al. (2015). A fast and adaptive algorithm for image restoration. IEEE Transactions on Circuits \& Systems I Fundamental Theory \& Applications, 46 (1),216220. 
[9] Meher PK, Sang YP. (2014). Critical-Path Analysis and Low-Complexity Implementation of the LMS Adaptive Algorithm. IEEE Transactions on Circuits \& Systems I Regular Papers, 61 (3),778-788.

[10] Thipphavong DP, Schultz CA, Lee AG, et al. (2015). Adaptive Algorithm to Improve Trajectory Prediction Accuracy of Climbing Aircraft. Journal of Guidance Control \& Dynamics, 36 (1),15-24.

[11] Di S, Wang CL, Cappello F. (2014). Adaptive Algorithm for Minimizing Cloud Task Length with Prediction Errors. IEEE Transactions on Cloud Computing, 2 (2),194-207.
[12] Xiao W, Yinfeng W, Ning Y, et al. (2012). A SelfAdaption Link-Quality Detection Algorithm for Data Collecting in OSN. Journal of Beijing University of Aeronautics \& Astronautics, 38 (8), 516-522.

[13] Jing X, Zhang J. (2015). An intelligent selfadaption complex event processing framework with dynamic context detection and automatic event pattern modification abilities. Journal of Intelligent \& Fuzzy Systems, 29 (5), 1739-1749.

[14] Liao J, Qi Q, Wang J, et al. A dual mode selfadaption handoff for multimedia services in mobile cloud computing environment. Multimedia Tools \& Applications, 75 (8), 1-26.

\section{INCDMTM INNOVATIVE PRODUCTS:}

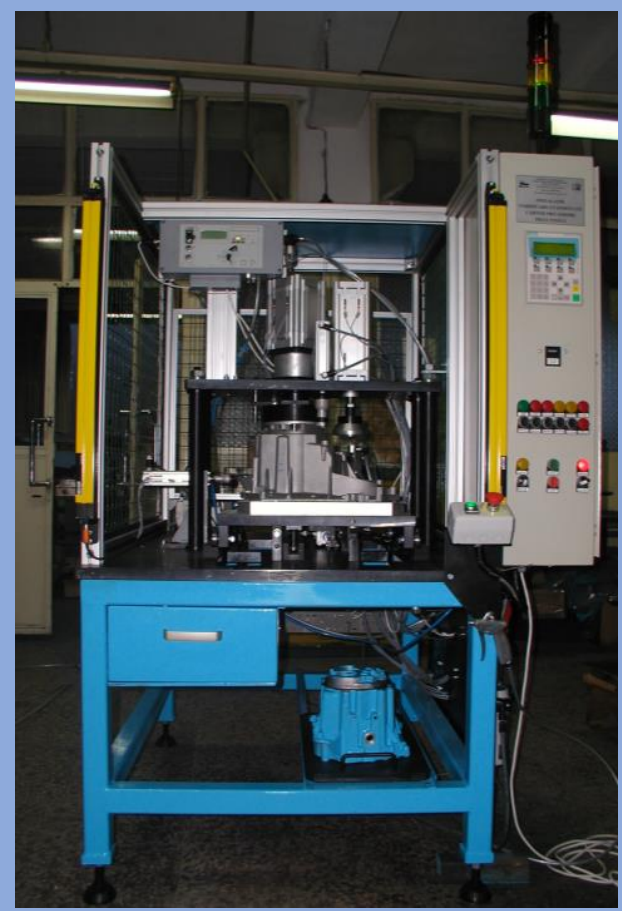

TIGHTNESS CONTROL EOUIPMENT FOR FINITE GEAR CASING 